\title{
The Size of a Formula as a Measure of Complexity
}

\author{
Lauri Hella \\ School of Information Sciences, Mathematics \\ University of Tampere \\ Jouko Väänänen* \\ Department of Mathematics and Statistics \\ University of Helsinki \\ and \\ Institute for Logic, Language and Computation \\ University of Amsterdam
}

\section{Introduction}

We propose a refinement of the usual Ehrenfeucht-Fraissé game. The new game will help us make finer distinctions than the traditional one. In particular, it can be used to measure not only quantifier rank but also lengths of conjunctions and disjunctions needed for expressing a given property. Our game is similar to the game in [1] and in [5].

The most common measure of complexity of a first order sentence is its quantifier rank, and the method of Ehrenfeucht-Fraïssé games can be successfully used to prove lower bound results for this measure of complexity. However, the number of non-equivalent first order formulas of quantifier rank $n$ is an exponential tower of height $n$ with a polynomial depending on the vocabulary on the top. Thus we are not very close to knowing the formula

\footnotetext{
* Research partially supported by grant 251557 of the Academy of Finland and the EUROCORES LogICCC LINT programme.
} 
if we merely know its quantifier rank. In this paper we measure complexity in terms of the number of symbols in the formula. The advantage of our measure is that there are only $2^{P(n)}$ non-equivalent formulas with $n$ symbols, where $P(x)$ is a polynomial depending on the vocabulary.

Let us consider the question of the complexity of deciding whether a binary string

$$
s_{1} s_{2} \ldots s_{n}=10110010 \ldots 01
$$

has a certain pattern. One approach to this is circuit complexity. For example, it is proved in [3] that constant depth circuits cannot decide the parity of (1), i.e. the question whether (1) has an even number of ones. Another approach is computational complexity, where the parity of (1) is easily decided in linear time. In so called descriptive complexity the question is posed, what is the logical complexity of the simplest formula in a given logic that expresses the property of (11) in question, for example parity. Concerning this type of problems, it was proved in [2] that the question whether a set $R$ of $n+1$-sequences

$$
\left(a_{1}, \ldots, a_{n+1}\right)
$$

from a finite set $\{1, \ldots, m\}$ has an even cardinality cannot be expressed in existential second order logic, where the second order variables range over $\leq n$-ary relations, that is, there is no sentence $\phi$ of such existential second order logic with the property that the structure $(\{1, \ldots, m\}, R)$ satisfies $\phi$ if and only if $R$ has even cardinality.

Let us return to (1). A natural setup of using logic to study the question of complexity of finding patterns in (1) is to use the propositional logic with propositional symbols

$$
p_{1}, p_{2}, \ldots, p_{n}
$$

with the meaning

$$
p_{i} \text { is true if and only if } s_{i}=1 \text {. }
$$

Propositional logic gives rise to a variety of complexity measures. We focus here on the simplest of them, namely the number of occurrences of propositional symbols in a formula. Up to a constant factor this is the same as the number of symbols, that is, the length of the formula.

The parity of (1) is naturally expressed by the propositional formula

$$
\bigvee\left\{\bigwedge_{i=1}^{n} p_{i}^{A}: A \subseteq\{1, \ldots, n\},|A| \text { even }\right\}
$$


where

$$
p_{i}^{A}= \begin{cases}p_{i} & \text { if } i \in A \\ \neg p_{i} & \text { if } i \notin A,\end{cases}
$$

which gives the upper bound $\frac{1}{2} n 2^{n}$ to the size of the smallest formula expressing parity. However, there is a shorter formula $\psi$ defined as follows: $\psi=\phi_{1, n}$, where for $1 \leq i<j \leq n$ and $k=\left\lfloor\frac{i+j}{2}\right\rfloor$

$$
\phi_{i, j}=\left(\phi_{i, k} \wedge \phi_{k+1, j}\right) \vee\left(\neg \phi_{i, k} \wedge \neg \phi_{k+1, j}\right)
$$

and

$$
\phi_{i, i}=\neg p_{i}
$$

This gives (up to a constant factor) the upper bound $n^{2}$ for the shortest propositional formula expressing parity 1 . Krapchenko (see [6, p 258]) has proved in 1971 that this is optimal. We prove below the same result using our refinement of the Ehrenfeucht-Fraïssé game.

For a slightly different complexity question, suppose we have n unary predicates:

$$
P_{1}(x), \ldots, P_{n}(x) .
$$

We ask what is the length of the shortest formula in first order logic with the unary predicates (7) that expresses the property that every Boolean combination of the predicates is non-empty. This property can be written as the existential sentence:

$$
\bigwedge\left\{\exists x \bigwedge_{i=1}^{n} P_{i}^{A}(x): A \subseteq\{1, \ldots, n\}\right\},
$$

where, as above in ([6), $P_{i}^{A}(x)=P_{i}(x)$ if $i \in A$, and $P_{i}^{A}(x)=\neg P_{i}(x)$ if $i \notin A$.

This gives the upper bound $(n+1) 2^{n}$ to the size of the smallest sentence expressing the given property (we define the size of a formula to be the total number of atomic subformulas and quantifiers occurring in it). In Section 5 , we show that this is optimal for existential sentences. If we give up existential sentences and allow a quantifier alternation, we have a smaller sentence $\phi$ defined as follows:

$$
\forall x \exists y \bigwedge_{i=1}^{n}\left(P_{i}(x) \leftrightarrow P_{i+1}(y)\right) \wedge
$$

\footnotetext{
${ }^{1}$ More precisely, a straightforward induction shows that the size of $\psi$ is at most $(n+1)^{2}$, and if $n$ is a power of 2 , then the size of $\psi$ is exactly $n^{2}$.
} 


$$
\forall x \exists y\left(\bigwedge_{i=2}^{n}\left(P_{i}(x) \leftrightarrow P_{i}(y)\right) \wedge\left(P_{1}(x) \leftrightarrow \neg P_{1}(y)\right)\right),
$$

where addition $i+1$ is to be calculated modulo $n$. The size of $\phi$ is only $8 n+4$, so this is really optimal (up to a constant factor) because we cannot have such a $\phi$ without mentioning each $P_{i}$ at least once.

In our final application, we consider the minimal size of a first-order sentence expressing that the length of a linear order is at least $n$. It is well known that this can be expressed by a sentence with quantifier rank $\lceil\log n\rceil+1$. Such a sentence $\phi_{n}$ can be obtained as follows: define first recursively formulas $\theta_{k}(x, y)$ by letting $\theta_{2}(x, y):=x<y$ and $\theta_{k}(x, y):=$ $\exists z\left(\theta_{l}(x, z) \wedge \theta_{m}(z, y)\right)$, where $l=\lfloor k / 2\rfloor+1$ and $m=\lceil k / 2\rceil$, for $k>2$. Thus, $\theta_{k}(x, y)$ says that there are at least $k$ elements in the interval $[x, y]$. Then $\phi_{n}$ can be defined as $\exists x \exists y \theta_{n}(x, y)$.

Clearly the size of $\phi_{n}$ is linear in $n$. However, with a clever trick of recycling quantified variables it is possible to define a variant $\phi_{n}^{\prime}$ of $\phi_{n}$ that has size $c\lceil\log n\rceil$, where $c$ is a small constant. Moreover, $\phi_{n}^{\prime}$ can be chosen to be in the 4-variable logic $\mathrm{FO}_{4}$ (see [4], Section 5). On the other hand, Grohe and Schweikardt prove in [4] that the minimal size of an equivalent sentence in $\mathrm{FO}_{3}$ is at least $\frac{1}{2} \sqrt{n}$. Their proof is based on a method which is related to the game we study in this paper, but it is not formulated in a game theoretic form.

\section{A Game for Propositional Logic}

We introduce now a game for measuring how long a propositional formula has to be to express a given property of binary strings

$$
s=s_{1} \ldots s_{n},
$$

where $s_{i} \in\{0,1\}$. We denote strings by $s, r, t$ etc. Let $n$ be the fixed length of the strings considered. We shall discuss nonempty sets $S$ of such strings and call them string properties. For example, $S$ could be the set of strings (9) where the cardinality of the set $\left\{i \in\{1, \ldots, n\}: s_{i}=1\right\}$ is even. Another example is the set of strings (9) with more ones than zeros. In an extreme case $S$ can be a singleton, e.g. the singleton constant one string $\{111 \ldots 1\}$.

Propositional formulas are built up from propositional symbols (3) by means of $\neg, \wedge$ and $\vee$. We use Greek letters $\phi, \psi$ etc to denote propositional 
formulas. The concept $s \models \phi$ of a string $s$ satisfying a propositional formula $\phi$ is defined in the usual way: $s \models p_{i}$ if $s_{i}=1 ; s \models \neg \phi$ if $s \not \models \phi ; s \models \phi \wedge \psi$ if $s \models \phi$ and $s \models \psi ; s \models \phi \vee \psi$ if $s \models \phi$ or $s \models \psi$.

Definition 1 Suppose $\phi$ is a propositional formula and $S$ a string property. We say that $\phi$ defines $S$ if for all strings $s$

$$
s \in S \Longleftrightarrow s \models \phi .
$$

We define the size $\mathrm{w}(\phi)$ of the propositional formula $\phi$ as follows:

$$
\begin{aligned}
\mathrm{w}\left(p_{i}\right) & =1 \\
\mathrm{w}(\neg \phi) & =\mathrm{w}(\phi) \\
\mathrm{w}(\phi \vee \psi) & =\mathrm{w}(\phi)+\mathrm{w}(\psi) \\
\mathrm{w}(\phi \wedge \psi) & =\mathrm{w}(\phi)+\mathrm{w}(\psi) .
\end{aligned}
$$

Note that $\mathrm{w}(\phi)$ is just the number of occurrences of propositional symbols in $\phi$. Up to a constant factor, this is the same as the number of symbols in $\phi$.

Note that while there are, up to logical equivalence, $2^{2^{n}}$ propositional formulas over the propositional symbols $p_{1}, \ldots, p_{n}$ altogether, there are for each $m$, up to logical equivalence, only at most $2^{m} \cdot(n+2)^{2 m}$ propositional formulas $\phi$ with $\mathrm{w}(\phi) \leq m$.

A natural question now is:

Question: Given a string property $S$, what is the size of the smallest propositional formula that defines $S$.

We shall define a game for the study of this question. In defining the game we follow mostly [1] and to a lesser degree [5]. This game resembles the usual Ehrenfeucht-Fraïssé game for the first order logic, but also the EhrenfeuchtFraïssé game for the independence friendly logic, presented in [5].

In the usual Ehrenfeucht-Fraïssé game we have two structures $\mathfrak{A}$ and $\mathfrak{B}$ and the game is about their similarity. Player II maintains that the structures are very similar. Player I maintains that there is a difference. During the game player I picks elements from either one of the models and player II tries to find similar elements in the other model.

In the new game we do not have just two models but instead two classes of models. Player II maintains that whatever features the models in one class have all in common, some member of the other class also has. The 
game to be defined is able to detect very minute differences in models and is in that sense finer than the usual Ehrenfeucht game. In particular, this game can detect differences in the size of conjunctions and disjunctions needed for separating the classes of models.

Definition 2 Let $S$ and $R$ be string properties and $w$ a positive integer. The game $\mathrm{EF}_{w}(S, R)$ has two players. The number $w$ is called the rank of the game. In the beginning the position is $(w, S, R)$. Suppose the position after $m$ moves is $\left(w_{m}, S_{m}, R_{m}\right)$. There are the following two possibilities for the continuation of the game:

Left splitting move: Player I first chooses numbers $u$ and $v$ such that $1 \leq$ $u, v<w$ and $u+v=w$. Then player I represents $S_{m}$ as a union $C \cup D$. Now the game continues from the position $\left(u, C, R_{m}\right)$ or from the position $\left(v, D, R_{m}\right)$, and player II can choose which.

Right splitting move: Player I first chooses numbers $u$ and $v$ such that $1 \leq u, v<w$ and $u+v=w$. Then Player I represents $R_{m}$ as a union $C \cup D$. Now the game continues from the position $\left(u, S_{m}, C\right)$ or from the position $\left(v, S_{m}, D\right)$, and player II can choose which.

The game ends in a position $\left(w_{m}, S_{m}, R_{m}\right)$ and player $I$ wins if there is a propositional symbol $p_{i}$ such that either $s \models p_{i}$ for all $s \in S_{m}$ and $r \forall p_{i}$ for all $r \in R_{m}$, or $s \forall p_{i}$ for all $s \in S_{m}$ and $r \models p_{i}$ for all $r \in R_{m}$. Player II wins the game if they reach a position $\left(w_{m}, S_{m}, R_{m}\right)$ such that $w_{m}=1$ and player I does not win in this position.

This is a game of perfect information and the concept of winning strategy is defined as usual. Since the rank $w_{m}$ decreases in each move, the game always ends in a finite number of moves. Hence the game is determined: exactly one of the players has a winning strategy in $\operatorname{EF}_{w}(S, R)$.

Note that if the players have reached a position $\left(w_{m}, S_{m}, R_{m}\right)$ in the game $\mathrm{EF}_{w}(S, R)$, then the continuation of the game from that position onwards can be seen as a play of the game $\mathrm{EF}_{w_{m}}\left(S_{m}, R_{m}\right)$. Thus, player I (player II) has a winning strategy in the game $\mathrm{EF}_{w}(S, R)$ from position $\left(w_{m}, S_{m}, R_{m}\right)$ onwards if an only if player I (player II, respectively) has a winning startegy in the game $\mathrm{EF}_{w_{m}}\left(S_{m}, R_{m}\right)$.

We say that a propositional formula $\phi$ separates string properties $S$ and $R$, in symbols $(S, R) \models \phi$, if $s \models \phi$ for all $s \in S$ and $r \not \models \phi$ for all $r \in R$. 
Theorem 3 Suppose $(S, R)$ is a pair of string properties, and let $w$ be a positive integer. Then the following conditions are equivalent:

$(1)_{w}$ Player I has a winning strategy in the game $\mathrm{EF}_{w}(S, R)$.

$(2)_{w}$ There is a propositional formula $\phi$ of size $\leq w$ such that $(S, R) \models \phi$.

Proof. We prove the equivalence of $(1)_{w}$ and $(2)_{w}$ by induction on $w$. Consider first the case $w=1$. By Definition 2, there are no moves in the game $\mathrm{EF}_{1}(S, R)$, and player I wins if and only if there is a proposition symbol $p_{i}$ such that either $(S, R) \models p_{i}$, or $(S, R) \models \neg p_{i}$. Since $\mathrm{w}\left(p_{i}\right)=\mathrm{w}\left(\neg p_{i}\right)=1$, we have $(1)_{1} \Longrightarrow(2)_{1}$. On the other hand, if $\mathrm{w}(\phi) \leq 1$, then $\phi$ is, up to logical equivalence, either a proposition symbol, or the negation of a proposition symbol. Thus, $(2)_{1}$ implies $(1)_{1}$.

Let us then consider the case $w>1$, and assume $(1)_{v} \Longleftrightarrow(2)_{v}$ for all $v<w$ as an induction hypothesis. To prove $(1)_{w} \Longleftrightarrow(2)_{w}$, assume first that player I has a winning strategy in the game $\operatorname{EF}_{w}(S, R)$. We have the following two cases according to the type of the first move in the winning strategy of player I.

Case 1: Player I makes a left splitting move by choosing $u, v, C$ and $D$ such that $1 \leq u, v<w, w=u+v$ and $S=C \cup D$. Since this move is given by a winning strategy, player I has a winning strategy in both of the games $\mathrm{EF}_{u}(C, R)$ and $\mathrm{EF}_{v}(D, R)$. By induction hypothesis, there are formulas $\psi$ and $\theta$ such that $\mathrm{w}(\psi) \leq u, \mathrm{w}(\theta) \leq v,(C, R) \models \psi$ and $(D, R) \models \theta$. Thus, $s \models \psi$ for all $s \in C$ and $s \models \theta$ for all $s \in D$, whence $s \models \psi \vee \theta$ for all $s \in S$. On the other hand, for all $r \in R, r \forall \forall \psi$ and $r \forall \forall$, whence consequently $r \not \vee \psi \vee \theta$. It follows that $(S, R) \models \psi \vee \theta$. As $\mathrm{w}(\psi \vee \theta)=\mathrm{w}(\psi)+\mathrm{w}(\theta) \leq$ $u+v=w,(2)_{w}$ is true.

Case 2: Player I makes a right splitting move by choosing $u, v, C$ and $D$ such that $1 \leq u, v<w, w=u+v$ and $R=C \cup D$. Since this move is given by a winning strategy, player I has a winning strategy in both of the games $\mathrm{EF}_{u}(S, C)$ and $\mathrm{EF}_{v}(S, D)$. By induction hypothesis there are formulas $\psi$ and $\theta$ such that $\mathrm{w}(\psi) \leq u, \mathrm{w}(\theta) \leq v,(S, C) \models \psi$ and $(S, D) \models \theta$. Thus, for all $s \in S, s \models \psi$ and $s \models \theta$, whence $s \models \psi \wedge \theta$. On the other hand, $r \not \models \psi$ for all $r \in C$ and $r \not \forall \theta$ for all $r \in D$, whence $r \not \neq \psi \wedge \theta$ for all $r \in R$. It follows that $(S, R) \models \psi \wedge \theta$. As $\mathrm{w}(\psi \wedge \theta)=\mathrm{w}(\psi)+\mathrm{w}(\theta) \leq u+v=w,(2)_{w}$ is true.

To prove the converse implication $(2)_{w} \Longrightarrow(1)_{w}$, assume that there is a formula $\phi$ of size $\leq w$ such that $(S, R) \models \phi$. We show that then player I 
has a winning strategy in the game $\mathrm{EF}_{w}(S, R)$. We assume without loss of generality that $\phi$ is in negation normal form 2 . The strategy depends on $\phi$ as follows.

Case 1: $\phi$ is a proposition symbol or the negation of a proposition symbol. Then by the definition of the game $\mathrm{EF}_{w}$, player I wins without making any moves.

Case 2: $\phi$ is $\psi \vee \theta$. Let $C$ be the set of strings $s \in S$ such that $s \models \psi$, and let $D$ be the set of strings $s \in S$ such that $s \models \theta$. Since $s \models \phi$ for every $s \in S$, we have $S=C \cup D$. Moreover, since $r \not \forall \phi$ for every $r \in R$, we have $(C, R) \models \psi$ and $(D, R) \models \theta$. Finally, as $\mathrm{w}(\phi) \leq w$, there are $u$ and $v$ such that $w=u+v, \mathrm{w}(\psi) \leq u$ and $\mathrm{w}(\theta) \leq v$. Note that $u, v<w$, and so, by induction hypothesis, player I has a winning strategy in the games $\operatorname{EF}_{u}(C, R)$ and $\operatorname{EF}_{v}(D, R)$. Thus, using $w=u+v, S=C \cup D$ as his first move and these winning strategies in the rest of the game, player I is guaranteed to win the game $\mathrm{EF}_{w}(S, R)$.

Case 3: $\phi$ is $\psi \wedge \theta$. Let $C$ be the set of strings $r \in R$ such that $r \not \neq \psi$, and let $D$ be the set of strings $r \in R$ such that $s \not \forall \theta$. Since $r \not \neq \phi$ for every $r \in R$, we have $R=C \cup D$. Moreover, since $s \models \phi$ for every $s \in S$, we have $(S, C) \models \psi$ and $(S, D) \models \theta$. Finally, as $\mathrm{w}(\phi) \leq w$, there are $u$ and $v$ such that $w=u+v, \mathrm{w}(\psi) \leq u$ and $\mathrm{w}(\theta) \leq v$. Note that $u, v<w$, and so, by induction hypothesis, player I has a winning strategy in the games $\operatorname{EF}_{u}(S, C)$ and $\operatorname{EF}_{v}(S, D)$. Thus, using $w=u+v, R=C \cup D$ in his first move, and these winning strategies in the rest of the game, player I is guaranteed to win the game $\mathrm{EF}_{w}(S, R)$.

Q.E.D.

Corollary 4 Suppose $K$ is a string property. Then the following conditions are equivalent:

(1) $K$ is definable by a propositional formula $\phi$ of size $\leq w$.

(2) Player I has a winning strategy in the game $\operatorname{EF}_{w}(S, R)$ for all $S$ and $R$ such that $S \subseteq K$ and $R \cap K=\emptyset$.

\footnotetext{
${ }^{2} \mathrm{~A}$ formula is in negation normal form, if all negations occurring in it are in front of proposition symbols. A straightforward induction shows that for any formula $\phi$ there is an equivalent formula $\phi^{\prime}$ in negation normal form such that $\mathrm{w}\left(\phi^{\prime}\right)=\mathrm{w}(\phi)$.
} 
Proof. Suppose $K$ is defined by $\phi$ of size $\leq w$. Let $S$ and $R$ be given so that $S \subseteq K$ and $R \cap \mathcal{K}=\emptyset$. Thus every model in $S$ satisfies $\phi$ and no model in $R$ does, whence $(S, R) \models \phi$. By Theorem 3 , player I has a winning strategy in $\mathrm{EF}_{w}(S, R)$. For the converse, suppose player I has a winning strategy in $\mathrm{EF}_{w}(S, R)$ for all $(S, R)$ with $S \subseteq \mathcal{K}$ and $R \cap K$. This holds in particular if $S$ is $K$ and $R$ is the complement of $K$. Thus for this choice of $S$ and $R$ we have $(S, R) \models \phi$ for some $\phi$ of size $\leq w$. Thus $K$ is defined by $\phi$. Q.E.D.

We get the following method for showing that a string property $K$ is not definable by a propositional formula of size $\leq w$ : We find classes $S$ and $R$ such that

1. $S \subseteq K$.

2. $R \cap K=\emptyset$.

3. Player II has a winning strategy in $\operatorname{EF}_{w}(S, R)$.

\section{The Propositional Complexity of Parity}

We shall now prove that the parity of a binary string of $n$ bits cannot be expressed with a propositional formula smaller than $n^{2}$. The result has been proved in [6] with a method which is very similar to ours, even if it does not explicitly use games. We present the proof as an example of the use of our game.

Suppose $S$ and $R$ are disjoint sets of binary strings of length $n$. Let

$$
E=\left\{(f, g) \in S \times R:\left|\left\{i: f_{i} \neq g_{i}\right\}\right|=1\right\} .
$$

The density of the pair $(S, R)$ is defined to be the pair

$$
D(S, R)=(s, r)
$$

where

$$
s=\frac{|E|}{|S|} \text {, and } r=\frac{|E|}{|R|} .
$$

Lemma 5 If $s>1$ or $r>1$, then there is no proposition symbol $p_{i}$ such that $(S, R) \models p_{i}$ or $(S, R) \models \neg p_{i}$. 
Proof. Suppose $s>1$ and $i \in\{1, \ldots, n\}$. Then there are $f \in S$ and $g, h \in R$ such that $(f, g),(f, h) \in E$. Clearly this means that $f_{i}=g_{i}$ or $f_{i}=h_{i}$, whence $f \models p_{i} \Longleftrightarrow g \models p_{i}$ or $f \models p_{i} \Longleftrightarrow h \models p_{i}$. Thus, if all strings in $S$ satisfy $p_{i}\left(\neg p_{i}\right)$, then there is a string in $R$ satisfying $p_{i}\left(\neg p_{i}\right.$, respectively). It follows that neither $p_{i}$, nor $\neg p_{i}$ separates the sets $S$ and $R$.

In the case $r>1$ there are $f, g \in S$ and $h \in R$ such that $(f, h),(g, h) \in E$. Then we have $h_{i}=f_{i}$ or $h_{i}=g_{i}$, whence $h \models p_{i} \Longleftrightarrow f \models p_{i}$ or $h \models p_{i} \Longleftrightarrow$ $g \models p_{i}$. As above, we conclude that neither $p_{i}$, nor $\neg p_{i}$ separates the sets $S$ and $R$.

Q.E.D.

Lemma 6 (a) Suppose $D(S, R)=(s, r)$ and $S=S_{0} \cup S_{1}$, where $S_{0} \cap S_{1}=\emptyset$. Let $D\left(S_{0}, R\right)=\left(s_{0}, r_{0}\right)$ and $D\left(S_{1}, R\right)=\left(s_{1}, r_{1}\right)$. Then $s_{0} r_{0}+s_{1} r_{1} \geq s r$.

(b) Suppose $D(S, R)=(s, r)$ and $R=R_{0} \cup R_{1}$, where $R_{0} \cap R_{1}=\emptyset$. Let $D\left(S, R_{0}\right)=\left(s_{0}, r_{0}\right)$ and $D\left(S, R_{1}\right)=\left(s_{1}, r_{1}\right)$. Then $s_{0} r_{0}+s_{1} r_{1} \geq s r$.

Proof. We prove (a); the proof of (b) is similar. Clearly $r_{0}+r_{1}=r$ and $\frac{r_{0}}{s_{0}}+\frac{r_{1}}{s}=\frac{r}{s}$. Since the harmonic mean is never greater than the arithmetic mean 3 , we have

$$
\frac{s_{0} r_{0}+s_{1} r_{1}}{r}=\frac{s_{0} r_{0}+s_{1} r_{1}}{r_{0}+r_{1}} \geq \frac{r_{0}+r_{1}}{\frac{r_{0}}{s_{0}}+\frac{r_{1}}{s_{1}}}=\frac{r}{\frac{r}{s}}=s,
$$

and the claim follows.

Q.E.D.

Lemma 7 Suppose $D(S, R)=(s, r)$ and $w<s r$. Then player II has a winning strategy in $\mathrm{EF}_{w}(S, R)$.

Proof. The proof is by induction on $w$. In the case $w=1$, the assumption $w<s r$ implies that either $s>1$ or $r>1$. By Lemma 5 , there is no proposition symbol $p_{i}$ such that $(S, R) \models p_{i}$ or $(S, R) \models \neg p_{i}$. Since the game $\mathrm{EF}_{1}(S, R)$ ends in its initial position $(1, S, R)$, this means that player II automatically wins this game.

Assume then that $w>1$. Using Lemma 5 again, we see that player I does not win the game $\operatorname{EF}_{w}(S, R)$ without making moves. Suppose then that player I makes a left splitting move $w=u+v$ and $S=C \cup D$. Let $S_{0} \subseteq C$ and $S_{1} \subseteq D$ be sets such that $S=S_{0} \cup S_{1}$ and $S_{0} \cap S_{1}=\emptyset$.

\footnotetext{
${ }^{3}$ We are indebted to Kerkko Luosto for pointing this out.
} 
Let $D\left(S_{0}, R\right)=\left(s_{0}, r_{0}\right)$ and $D\left(S_{1}, R\right)=\left(s_{1}, r_{1}\right)$. Then by Lemma 6(a), $s_{0} r_{0}+s_{1} r_{1} \geq s r$. Thus $s_{0} r_{0}>u$ or $s_{1} r_{1}>v$, for otherwise

$$
s r \leq s_{0} r_{0}+s_{1} r_{1} \leq u+v=w
$$

contrary to the assumption. If $s_{0} r_{0}>u$, then by induction hypothesis, player II has a winning strategy in the game $\operatorname{EF}_{u}\left(S_{0}, R\right)$, whence, a fortiori, he has a winning strategy in $\operatorname{EF}_{u}(C, R)$. Otherwise, $s_{1} r_{1}>v$, and by the same argument, player II has a winning strategy in the game $\mathrm{EF}_{v}(D, R)$. Thus, in any case, player II can make his move in such a way that he is guaranteed to win.

The case of a right splitting move $w=u+v$ and $R=C \cup D$ is proved in the same way by using Lemma 6(b).

We are now ready to prove the promised lower bound for parity of binary strings.

Corollary 8 ([6]) If $\phi$ is a propositional formula expressing the parity of strings $s \in\{0,1\}^{n}$, then the size of $\phi$ is at least $n^{2}$.

Proof. Let $S$ be the set of all strings $f \in\{0,1\}^{n}$ such that $\mid\left\{i: f_{i}=\right.$ $1\} \mid$ is even, and let $R$ be the complement of $S$. Thus, $|S|=|R|=2^{n-1}$. Furthemore, for each string $f \in S$, there are $n$ different strings $g \in\{0,1\}^{n}$ such that $\left|\left\{i: f_{i} \neq g_{i}\right\}\right|=1$, and all these strings are in $R$. Thus, we have $|E|=2^{n-1} n$, and consequently $D(S, R)=(n, n)$. By Lemma 7, player II has a winning strategy in the game $\operatorname{EF}_{w}(S, R)$ whenever $w<n^{2}$, and we conclude that $S$ is not definable by any formula of size less than $n^{2}$. Q.E.D.

As noted in the introduction, the parity of strings in $\{0,1\}^{n}$ can be expressed by a formula of size at most $(n+1)^{2}$. Thus, the lower bound $n^{2}$ cannot be essentially improved.

\section{A Game for Predicate Logic}

We shall next define a game that can be used for measuring the size of a first-order sentence needed for expressing properties of models. For the sake of simplicity, we will only consider models with relational vocabulary.

We need to fix some notation first. The universe of a model $\mathfrak{A}$ is denoted by $A$, of $\mathfrak{B}$ by $B$, etc. We use $x_{j}, j \in \mathbb{N}$, to denote variables. A variable 
assignment for a model $\mathfrak{A}$ is a finite partial mapping $\alpha: \mathbb{N} \rightarrow A$. The finite domain of $\alpha$ is denoted by $\operatorname{dom}(\alpha)$. If $\phi$ is a formula, then $(\mathfrak{A}, \alpha) \models \phi$ means that the assignment $\alpha$ satisfies the formula $\phi$ in the model $\mathfrak{A}$. For this to be meaningful the domain of the assignment $\alpha$ has to include all the $j$ for which the variable $x_{j}$ is free in $\phi$.

We shall discuss classes $\mathcal{A}$ of structures $(\mathfrak{A}, \alpha)$, where $\mathfrak{A}$ is a model and $\alpha$ is an assignment. We assume that whenever $(\mathfrak{A}, \alpha),(\mathfrak{B}, \beta) \in \mathcal{A}$, then $\mathfrak{A}$ and $\mathfrak{B}$ have the same vocabulary, and $\alpha$ and $\beta$ have the same domain, which we denote by $\operatorname{dom}(\mathcal{A})$. If $\alpha$ is an assignment on $\mathfrak{A}, a \in A$ and $j \in \mathbb{N}$, then $\alpha(a / j)$ is the assignment that maps $j$ to $a$ and agrees with $\alpha$ otherwise. If $F$ is a choice function on $\mathcal{A}$, that is, $F$ is a function defined on $\mathcal{A}$ such that $F((\mathfrak{A}, \alpha)) \in A$ for all $(\mathfrak{A}, \alpha) \in \mathcal{A}$, then $\mathcal{A}(F / j)$ is defined as $\{(\mathfrak{A}, \alpha(F((\mathfrak{A}, \alpha)) / j):(\mathfrak{A}, \alpha) \in$ $\mathcal{A}\}$. Finally, $\mathcal{A}(\star / j)=\{(\mathfrak{A}, \alpha(a / j)):(\mathfrak{A}, \alpha) \in \mathcal{A}, a \in A\}$.

Let $\mathcal{A}$ and $\mathcal{B}$ be classes of structures of a fixed relational vocabulary. Assume further that $\operatorname{dom}(\mathcal{A})=\operatorname{dom}(\mathcal{B})$, and $\phi$ is a formula such that $j \in$ $\operatorname{dom}(\mathcal{A})$ for all variables $x_{j}$ which are free in $\phi$. As in the case of propositional logic, we say that $\phi$ separates the classes $\mathcal{A}$ and $\mathcal{B},(\mathcal{A}, \mathcal{B}) \models \phi$, if $(\mathfrak{A}, \alpha) \models \phi$ for all $(\mathfrak{A}, \alpha) \in \mathcal{A}$ and $(\mathfrak{B}, \beta) \not \neq \phi$ for all $(\mathfrak{B}, \beta) \in \mathcal{B}$.

Definition 9 Let $\mathcal{A}$ and $\mathcal{B}$ be classes of structures of the same relational vocabulary with $\operatorname{dom}(\mathcal{A})=\operatorname{dom}(\mathcal{B})$, and let $w$ be a positive integer. The game $\mathrm{EF}_{w}(\mathcal{A}, \mathcal{B})$ has two players. The number $w$ is called the rank of the game. In the beginning the position is $(w, \mathcal{A}, \mathcal{B})$. Suppose the position after $m$ moves is $\left(w_{m}, \mathcal{A}_{m}, \mathcal{B}_{m}\right)$, where $\operatorname{dom}\left(\mathcal{A}_{m}\right)=\operatorname{dom}\left(\mathcal{B}_{m}\right)$. There are the following four possibilities for the continuation of the game:

Left splitting move: Player I first chooses numbers $u$ and $v$ such that $1 \leq$ $u, v<w$ and $u+v=w_{m}$. Then Player I represents $\mathcal{A}_{m}$ as a union $\mathcal{C} \cup \mathcal{D}$. Now the game continues from the position $\left(u, \mathcal{C}, \mathcal{B}_{m}\right)$ or from the position $\left(v, \mathcal{D}, \mathcal{B}_{m}\right)$, and player II can choose which.

Right splitting move: Player I first chooses numbers $u$ and $v$ such that $1 \leq u, v<w$ and $u+v=w_{m}$. Then Player I represents $\mathcal{B}_{m}$ as a union $\mathcal{C} \cup \mathcal{D}$. Now the game continues from the position $\left(u, \mathcal{A}_{m}, \mathcal{C}\right)$ or from the position $\left(v, \mathcal{A}_{m}, \mathcal{D}\right)$, and player II can choose which.

Left supplementing move: Player $I$ chooses a natural number $j$ and a choice function $F$ for $\mathcal{A}_{m}$. Then the game continues from the position $\left(w_{m}-1, \mathcal{A}_{m}(F / j), \mathcal{B}_{m}(\star / j)\right)$. 
Right supplementing move: Player I chooses a natural number $j$ and a choice function $F$ for $\mathcal{B}_{m}$. Then the game continues from the position $\left(w_{m}-1, \mathcal{A}_{m}(\star / j), \mathcal{B}_{m}(F / j)\right)$.

The game ends in a position $\left(w_{m}, \mathcal{A}_{m}, \mathcal{B}_{m}\right)$ and player I wins if there is an atomic or a negated atomic formula $\phi$ such that $\left(\mathcal{A}_{m}, \mathcal{B}_{m}\right) \models \phi$. Player II wins the game if they reach a position $\left(w_{m}, S_{m}, R_{m}\right)$ such that $w_{m}=1$ and player I does not win in this position.

This is a game of perfect information and the concept of winning strategy is defined as usual. The game is determined by the Gale-Stewart theorem.

We define the size $\mathrm{w}(\phi)$ of the formula $\phi$ of predicate logic as follows:

$$
\begin{aligned}
\mathrm{w}(\phi) & =1 \text { for atomic } \phi \\
\mathrm{w}(\neg \phi) & =\mathrm{w}(\phi) \\
\mathrm{w}(\phi \vee \psi) & =\mathrm{w}(\phi)+\mathrm{w}(\psi) \\
\mathrm{w}(\phi \wedge \psi) & =\mathrm{w}(\phi)+\mathrm{w}(\psi) \\
\mathrm{w}\left(\exists x_{j} \phi\right) & =\mathrm{w}(\phi)+1 \\
\mathrm{w}\left(\forall x_{j} \phi\right) & =\mathrm{w}(\phi)+1
\end{aligned}
$$

Note that there are for each $w$, up to logical equivalence, only finitely many formulas of size $\leq w$.

Theorem 10 Suppose $(\mathcal{A}, \mathcal{B})$ is a pair of classes of structures of the same vocabulary, and let $w$ be a positive integer. Then the following conditions are equivalent:

$(1)_{w}$ Player I has a winning strategy in the game $\operatorname{EF}_{w}(\mathcal{A}, \mathcal{B})$.

$(2)_{w}$ There is a formula $\phi$ of predicate logic of size $\leq w$ such that $(\mathcal{A}, \mathcal{B}) \models \phi$.

Proof. We prove the equivalence of $(1)_{w}$ and $(2)_{w}$ by induction on $w$. Consider first the case $w=1$. By Definition 9, there are no moves in the game $\operatorname{EF}_{1}(\mathcal{A}, \mathcal{B})$, and player I wins if and only if there is an atomic formula $\phi$ such that either $(\mathcal{A}, \mathcal{B}) \models \phi$ or $(\mathcal{A}, \mathcal{B}) \models \neg \phi$. Since $\mathrm{w}(\phi)=\mathrm{w}(\neg \phi)=1$, we have $(1)_{1} \Longrightarrow(2)_{1}$. On the other hand, if $\mathrm{w}(\phi)=1$, then $\phi$ is, up to logical equivalence, either an atomic formula, or the negation of an atomic formula. Thus, $(2)_{1}$ implies $(1)_{1}$. 
Let us then consider the case $w>1$, and assume $(1)_{v} \Longleftrightarrow(2)_{v}$ for all $v<w$ as an induction hypothesis. To prove $(1)_{w} \Longleftrightarrow(2)_{w}$, assume first that player I has a winning strategy in the game $\operatorname{EF}_{w}(\mathcal{A}, \mathcal{B})$. We have the following four cases according to the type of the first move in the winning strategy of player I.

Case 1: Player I makes a left splitting move by choosing $u, v, \mathcal{C}$ and $\mathcal{D}$ such that $1 \leq u, v<w, w=u+v$ and $\mathcal{A}=\mathcal{C} \cup \mathcal{D}$. Since this move is given by a winning strategy, player I has a winning startegy in both of the games $\mathrm{EF}_{u}(\mathcal{C}, \mathcal{B})$ and $\mathrm{EF}_{v}(\mathcal{D}, \mathcal{B})$. By induction hypothesis, there are formulas $\psi$ and $\theta$ such that $\mathrm{w}(\psi) \leq u, \mathrm{w}(\theta) \leq v,(\mathcal{C}, \mathcal{B}) \models \psi$ and $(\mathcal{D}, \mathcal{B}) \models \theta$. Thus, $(\mathfrak{A}, \alpha)=\psi$ for all $(\mathfrak{A}, \alpha) \in \mathcal{C}$ and $(\mathfrak{A}, \alpha) \models \theta$ for all $(\mathfrak{A}, \alpha) \in \mathcal{D}$, whence $(\mathfrak{A}, \alpha)=\psi \vee \theta$ for all $(\mathfrak{A}, \alpha) \in \mathcal{A}$. On the other hand, for all $(\mathfrak{B}, \beta) \in \mathcal{B}$, $(\mathfrak{B}, \beta) \forall \forall \psi$ and $(\mathfrak{B}, \beta) \forall \forall$, whence consequently $(\mathfrak{B}, \beta) \forall \forall \psi \vee \theta$. It follows that $(\mathcal{A}, \mathcal{B}) \models \psi \vee \theta$. As $\mathrm{w}(\psi \vee \theta)=\mathrm{w}(\psi)+\mathrm{w}(\theta) \leq u+v=w,(2)_{w}$ is true.

Case 2: Player I makes a right splitting move by choosing $u, v, \mathcal{C}$ and $\mathcal{D}$ such that $1 \leq u, v<w, w=u+v$ and $\mathcal{B}=\mathcal{C} \cup \mathcal{D}$. Since this move is given by a winning strategy, player I has a winning startegy in both of the games $\operatorname{EF}_{u}(\mathcal{A}, \mathcal{C})$ and $\operatorname{EF}_{v}(\mathcal{A}, \mathcal{D})$. By induction hypothesis there are formulas $\psi$ and $\theta$ such that $\mathrm{w}(\psi) \leq u, \mathrm{w}(\theta) \leq v,(\mathcal{A}, \mathcal{C}) \models \psi$ and $(\mathcal{A}, \mathcal{D}) \models \theta$. Thus, for all $(\mathfrak{A}, \alpha) \in \mathcal{A},(\mathfrak{A}, \alpha) \models \psi$ and $(\mathfrak{A}, \alpha) \models \theta$, whence $(\mathfrak{A}, \alpha) \models \psi \wedge \theta$. On the other hand, $(\mathfrak{B}, \beta) \not \models \psi$ for all $(\mathfrak{B}, \beta) \in \mathcal{C}$ and $(\mathfrak{B}, \beta) \not \forall \theta$ for all $(\mathfrak{B}, \beta) \in \mathcal{D}$, whence $(\mathfrak{B}, \beta) \forall \psi \wedge \theta$ for all $(\mathfrak{B}, \beta) \in \mathcal{B}$. It follows that $(\mathcal{A}, \mathcal{B}) \models \psi \wedge \theta$. As $\mathrm{w}(\psi \wedge \theta)=\mathrm{w}(\psi)+\mathrm{w}(\theta) \leq u+v=w,(2)_{w}$ is true.

Case 3: Player I makes a left supplementing move by choosing a natural number $j$ and a choice function $F$ for $\mathcal{A}$. The next position in the game is then $(w-1, \mathcal{A}(F / j), \mathcal{B}(\star / j))$. Since this move is given by a winning strategy, player I has a winning strategy in the game $\mathrm{EF}_{w-1}(\mathcal{A}(F / j), \mathcal{B}(\star / j))$. By induction hypothesis there is a formula $\psi$ such that $\mathrm{w}(\psi) \leq w-1$ and $(\mathcal{A}(F / j), \mathcal{B}(\star / j)) \models \psi$. Let $\phi$ be the formula $\exists x_{j} \psi$. Then $\mathrm{w}(\phi)=\mathrm{w}(\psi)+1 \leq$ $w$, and it suffices to show that $(\mathcal{A}, \mathcal{B}) \models \phi$. Note first that for all $(\mathfrak{A}, \alpha) \in \mathcal{A}$, $(\mathfrak{A}, \alpha(a / j)) \models \psi$, where $a=F((\mathfrak{A}, \alpha))$. Thus we have $(\mathfrak{A}, \alpha) \models \phi$ for all $(\mathfrak{A}, \alpha) \in \mathcal{A}$. On the other hand, for all $(\mathfrak{B}, \beta) \in \mathcal{B}$ and all $b \in B$, we have $(\mathfrak{B}, \beta(b / j)) \not=\psi$, whence $(\mathfrak{B}, \beta) \not \models \phi$.

Case 4: Player I makes a right supplementing move by choosing a natural number $j$ and a choice function $F$ for $\mathcal{B}$. The next position in the game is then $(w-1, \mathcal{A}(\star / j), \mathcal{B}(F / j))$. Since this move is given by a winning strategy, 
player I has a winning strategy in the game $\mathrm{EF}_{w-1}(\mathcal{A}(\star / j), \mathcal{B}(F / j))$. By induction hypothesis there is a formula $\psi$ such that $\mathrm{w}(\psi) \leq w-1$ and $(\mathcal{A}(\star / j), \mathcal{B}(F / j))=\psi$. Let $\phi$ be the formula $\forall x_{j} \psi$. Then $\mathrm{w}(\phi)=\mathrm{w}(\psi)+1 \leq$ $w$, and it suffices to show that $(\mathcal{A}, \mathcal{B}) \models \phi$. Note first that for all $(\mathfrak{A}, \alpha) \in \mathcal{A}$ and all $a \in A$, we have $(\mathfrak{A}, \alpha(a / j)) \models \psi$. Thus we have $(\mathfrak{A}, \alpha) \models \phi$ for all $(\mathfrak{A}, \alpha) \in \mathcal{A}$. On the other hand, for all $(\mathfrak{B}, \beta) \in \mathcal{B},(\mathfrak{B}, \beta(b / j)) \forall \psi$, where $b=F((\mathfrak{B}, \beta))$. Thus we have $(\mathfrak{B}, \beta) \not \models \phi$ for all $(\mathfrak{B}, \beta) \in \mathcal{B}$.

To prove the converse implication $(2)_{w} \Longrightarrow(1)_{w}$, assume that there is a formula $\phi$ of size $\leq w$ such that $(\mathcal{A}, \mathcal{B}) \models \phi$. We show that then player I has a winning strategy in the game $\operatorname{EF}_{w}(\mathcal{A}, \mathcal{B})$. As in the case of propositional logic, we can assume without loss of generality that $\phi$ is in negation normal form. The strategy of player I depends on $\phi$ as follows.

Case 1: $\phi$ is an atomic formula or the negation of an atomic formula. Then by Definition 9, player I wins the game $\operatorname{EF}_{w}(\mathcal{A}, \mathcal{B})$ without making any moves.

Case 2: $\phi$ is $\psi \vee \theta$. Let $\mathcal{C}$ be the class of structures $(\mathfrak{A}, \alpha) \in \mathcal{A}$ such that $(\mathfrak{A}, \alpha) \models \psi$, and let $\mathcal{D}$ be the class of structures $(\mathfrak{A}, \alpha) \in \mathcal{A}$ such that $(\mathfrak{A}, \alpha)=\theta$. Since $(\mathfrak{A}, \alpha) \models \phi$ for every $(\mathfrak{A}, \alpha) \in \mathcal{A}$, we have $\mathcal{A}=\mathcal{C} \cup \mathcal{D}$. Moreover, since $(\mathfrak{B}, \beta) \not \models \phi$ for every $(\mathfrak{B}, \beta) \in \mathcal{B}$, we have $(\mathcal{C}, \mathcal{B}) \models \psi$ and $(\mathcal{D}, \mathcal{B})=\theta$. Finally, as $\mathrm{w}(\phi) \leq w$, there are $u$ and $v$ such that $w=u+v$, $\mathrm{w}(\psi) \leq u$ and $\mathrm{w}(\theta) \leq v$. Note that $u, v<w$, and so, by induction hypothesis, player I has a winning strategy in the games $\operatorname{EF}_{u}(\mathcal{C}, \mathcal{B})$ and $\operatorname{EF}_{v}(\mathcal{D}, \mathcal{B})$. Thus, using $w=u+v, \mathcal{A}=\mathcal{C} \cup \mathcal{D}$ as his first move and these winning strategies in the rest of the game, player $\mathrm{I}$ is guaranteed to win the game $\operatorname{EF}_{w}(\mathcal{A}, \mathcal{B})$.

Case 3: $\phi$ is $\psi \wedge \theta$. Let $\mathcal{C}$ be the class of structures $(\mathfrak{B}, \beta) \in \mathcal{B}$ such that $(\mathfrak{B}, \beta) \forall \psi$, and let $\mathcal{D}$ be the class of structures $(\mathfrak{B}, \beta) \in \mathcal{B}$ such that $(\mathfrak{B}, \beta) \not \forall \theta$. Since $(\mathfrak{B}, \beta) \not \models \phi$ for every $(\mathfrak{B}, \beta) \in \mathcal{B}$, we have $\mathcal{B}=\mathcal{C} \cup \mathcal{D}$. Moreover, since $(\mathfrak{A}, \alpha)=\phi$ for every $(\mathfrak{A}, \alpha) \in \mathcal{A}$, we have $(\mathcal{A}, \mathcal{C})=\psi$ and $(\mathcal{A}, \mathcal{D}) \models \theta$. Finally, as $\mathrm{w}(\phi) \leq w$, there are $u$ and $v$ such that $w=u+v$, $\mathrm{w}(\psi) \leq u$ and $\mathrm{w}(\theta) \leq v$. Note that $u, v<w$, and so, by induction hypothesis, player I has a winning strategy in the games $\operatorname{EF}_{u}(\mathcal{A}, \mathcal{C})$ and $\operatorname{EF}_{v}(\mathcal{A}, \mathcal{D})$. Thus, using $w=u+v, \mathcal{A}=\mathcal{C} \cup \mathcal{D}$ in his first move, and these winning strategies in the rest of the game, player I is guaranteed to win the game $\operatorname{EF}_{w}(\mathcal{A}, \mathcal{B})$.

Case 4: $\phi$ is $\exists x_{j} \psi$. Since $(\mathfrak{A}, \alpha) \models \phi$ for every $(\mathfrak{A}, \alpha) \in \mathcal{A}$, there is a choice function $F$ for $\mathcal{A}$ such that $(\mathfrak{A}, \alpha(F((\mathfrak{A}, \alpha)) / j)) \models \psi$ for all $(\mathfrak{A}, \alpha) \in \mathcal{A}$. Thus, $\left(\mathfrak{A}, \alpha^{*}\right) \models \psi$ for every $\left(\mathfrak{A}, \alpha^{*}\right) \in \mathcal{A}(F / j)$. On the other hand, for all $(\mathfrak{B}, \beta) \in \mathcal{B}$ we have $(\mathfrak{B}, \beta) \not \forall \phi$, whence $(\mathfrak{B}, \beta(b / j)) \forall \psi$ for all $b \in B$. 
In other words, $\left(\mathfrak{B}, \beta^{*}\right) \not \forall \psi$ for all $\left(\mathfrak{B}, \beta^{*}\right) \in \mathcal{B}(\star / j)$. Thus we conclude that $(\mathcal{A}(F / j), \mathcal{B}(\star / j))=\psi$. Note that $\mathrm{w}(\psi)=\mathrm{w}(\phi)-1 \leq w-1$, and so, by induction hypothesis, player I has a winning strategy in the game $\operatorname{EF}_{w-1}(\mathcal{A}(F / j), \mathcal{B}(\star / j))$. Thus, starting with the left supplementing move $j$ and $F$, and using this winning strategy in the rest of the game, player $\mathrm{I}$ is guaranteed to win the game $\operatorname{EF}_{w}(\mathcal{A}, \mathcal{B})$.

Case 5: $\phi$ is $\forall x_{j} \psi$. Since $(\mathfrak{B}, \beta) \not \models \phi$ for every $(\mathfrak{B}, \beta) \in \mathcal{B}$, there is a choice function $F$ for $\mathcal{B}$ such that $(\mathfrak{B}, \beta(F((\mathfrak{B}, \beta)) / j)) \forall \psi$ for all $(\mathfrak{B}, \beta) \in \mathcal{B}$. Thus, $\left(\mathfrak{B}, \beta^{*}\right) \not \forall \psi$ for every $\left(\mathfrak{B}, \beta^{*}\right) \in \mathcal{B}(F / j)$. On the other hand, for all $(\mathfrak{A}, \alpha) \in \mathcal{A}$ we have $(\mathfrak{A}, \alpha) \models \phi$, whence $(\mathfrak{A}, \alpha(a / j)) \models \psi$ for all $a \in A$. In other words, $\left(\mathfrak{A}, \alpha^{*}\right) \models \psi$ for all $\left(\mathfrak{A}, \alpha^{*}\right) \in \mathcal{A}(\star / j)$. Thus we conclude that $(\mathcal{A}(\star / j), \mathcal{B}(F / j)) \models \psi$. Note that $\mathrm{w}(\psi)=\mathrm{w}(\phi)-1 \leq w-1$, and so, by induction hypothesis, player I has a winning strategy in the game $\mathrm{EF}_{w-1}(\mathcal{A}(\star / j), \mathcal{B}(F / j))$. Thus, starting with the right supplementing move $j$ and $F$, and using this winning strategy in the rest of the game, player I is guaranteed to win the game $\operatorname{EF}_{w}(\mathcal{A}, \mathcal{B})$.

Q.E.D.

Corollary 11 Suppose $\mathcal{K}$ is a class of models of the same vocabulary. Then the following conditions are equivalent:

(1) $\mathcal{K}$ is the class of models of a first order sentence $\phi$ of size $\leq w$.

(2) Player I has a winning strategy in the game $\operatorname{EF}_{w}(\mathcal{A}, \mathcal{B})$ for all $\mathcal{A}$ and $\mathcal{B}$ such that $\mathcal{A} \subseteq \mathcal{K}$ and $\mathcal{B} \cap \mathcal{K}=\emptyset$.

Proof. Suppose $\mathcal{K}$ is the class of models of $\phi$ and $\mathrm{w}(\phi) \leq w$. Let $\mathcal{A}$ and $\mathcal{B}$ be given so that $\mathcal{A} \subseteq \mathcal{K}$ and $\mathcal{B} \cap \mathcal{K}=\emptyset$. Thus every model in $\mathcal{A}$ satisfies $\phi$ and no model in $\mathcal{B}$ does, whence $(\mathcal{A}, \mathcal{B}) \models \phi$. By Theorem 10 , player I has a winning strategy in $\operatorname{EF}_{w}(\mathcal{A}, \mathcal{B})$. For the converse, suppose player I has a winning strategy in $\operatorname{EF}_{w}(\mathcal{A}, \mathcal{B})$ for all $(\mathcal{A}, \mathcal{B})$ with $\mathcal{A} \subseteq \mathcal{K}$ and $\mathcal{B} \cap \mathcal{K}$. This holds in particular if $\mathcal{A}=\mathcal{K}$ and $\mathcal{B}$ is the complement of $\mathcal{K}$. Thus for this choice of $\mathcal{A}$ and $\mathcal{B}$ we have $(\mathcal{A}, \mathcal{B}) \models \phi$ for some $\phi$ of size $\leq w$. Thus $\mathcal{K}$ is the class of all models of $\phi$.

Q.E.D.

We get the following method for showing that a model class $\mathcal{K}$ is not definable by a first order sentence of size $\leq w$ : We find classes $\mathcal{A}$ and $\mathcal{B}$ such that

1. $\mathcal{A} \subseteq \mathcal{K}$. 
2. $\mathcal{B} \cap \mathcal{K}=\emptyset$.

3. Player II has a winning strategy in $\operatorname{EF}_{w}(\mathcal{A}, \mathcal{B})$.

\section{A Game for Existential Formulas}

The game $\mathrm{EF}_{w}$ can be used for solving questions of the type

Given a property of $\mathcal{P}$ models, what is the size of the smallest first order formula that defines $\mathcal{P}$ ?

But in some applications we are interested in definability by some restricted type of of formulas, rather than arbitrary first order formulas. For example, if a property $\mathcal{P}$ of models is known to be definable by an existential formula, it is natural to ask, what is the size of the smallest existential formula defining $\mathcal{P}$. We will now define a variant of the game $\mathrm{EF}_{w}$ that can be used in studying this question.

Here we say that a first order formula is existential if it is in negation normal form, and it does not contain any universal quantifiers. In other words, existential formulas are built from atomic formulas and negations of atomic formulas by using the connectives $\vee, \wedge$ and the quantifier $\exists$.

Intuitively, in the definition of the game $\mathrm{EF}_{w}$, left and right splitting moves correspond to the connectives $\vee$ and $\wedge$, while left and right supplementing moves correspond to the quantifiers $\exists$ and $\forall$. Thus, we obtain a game for existential formulas simply by dropping right supplementing moves.

Definition 12 Let $\mathcal{A}$ and $\mathcal{B}$ be classes of structures of the same relational vocabulary with $\operatorname{dom}(\mathcal{A})=\operatorname{dom}(\mathcal{B})$, and let $w$ be a positive integer. The existential game $\operatorname{EF}_{w}^{\exists}(\mathcal{A}, \mathcal{B})$ has the same rules as $\operatorname{EF}_{w}(\mathcal{A}, \mathcal{B})$, except that player $I$ is not allowed to make right supplementing moves.

Theorem 13 Suppose $(\mathcal{A}, \mathcal{B})$ is a pair of classes of structures of the same vocabulary, and let $w$ be a positive integer. Then the following conditions are equivalent:

$(1)_{w}$ Player I has a winning strategy in the game $\operatorname{EF}_{w}^{\exists}(\mathcal{A}, \mathcal{B})$.

$(2)_{w}$ There is an existential formula $\phi$ of size $\leq w$ such that $(\mathcal{A}, \mathcal{B}) \models \phi$. 
Proof. The claim is proved by a straightforward adaptation of the proof of Lemma 10. In the proof of $(1)_{w} \Longrightarrow(2)_{w}$ it suffices to note that omitting Case 4 , the formula $\phi$ separating the classes $\mathcal{A}$ and $\mathcal{B}$ will always be existential. Similarly, in the proof of the opposite implication, Case 5 never occurs as the separating formula $\phi$ is existential, whence the winning strategy of player I does not use right supplementing moves.

Q.E.D.

As in the case of the full first order game $\mathrm{EF}_{w}$, we get again a method for showing that a model class $\mathcal{K}$ is not definable by an existential formula of size $\leq w$ : We find classes $\mathcal{A}$ and $\mathcal{B}$ such that

1. $\mathcal{A} \subseteq \mathcal{K}$.

2. $\mathcal{B} \cap \mathcal{K}=\emptyset$.

3. Player II has a winning strategy in $\operatorname{EF}_{w}^{\exists}(\mathcal{A}, \mathcal{B})$.

\section{The Existential Complexity of Non-Empti- ness of Boolean Combinations}

We observed in the introduction that non-emptiness of all Boolean combinations of $n$ unary predicates can be defined with a sentence of size $8 n+4$ if a quantifier alternation is allowed. If only existential sentences are allowed, this can be expressed with a sentence of size $(n+1) 2^{n}$. We use now our game for existential formulas to show that $(n+1) 2^{n}$ is the best possible value.

For each binary string $s \in\{0,1\}^{n}$, let $\mathfrak{B}_{s}$ be the $\left\{P_{1}, \ldots, P_{n}\right\}$-structure such that for each $r \in\{0,1\}^{n}$, the Boolean combination of $P_{1}^{\mathfrak{B}_{s}}, \ldots, P_{n}^{\mathfrak{B}_{s}}$ corresponding to $r$ contains exactly two elements, $b_{r}$ and $c_{r}$, except that the combination corresponding to $s$ is empty. Furthermore, let $\mathfrak{A}$ be the structure in which each Boolean combination contains exactly one element; let $a_{r}$ be the element in the Boolean combination corresponding to $r \in\{0,1\}^{n}$. Let $\mathcal{A}_{0}$ be the class $\{(\mathfrak{A}, \emptyset)\}$, and let $\mathcal{B}_{0}$ be the class $\left\{\left(\mathfrak{B}_{s}, \emptyset\right): s \in\{0,1\}^{n}\right\}$. We will show that player II has a winning strategy in the existential game $\operatorname{EF}_{w}^{\exists}\left(\mathcal{A}_{0}, \mathcal{B}_{0}\right)$ for all $w<(n+1) 2^{n}$.

Since the game is existential, in any position $(u, \mathcal{A}, \mathcal{B})$, the set $\mathcal{A}$ contains only one structure $(\mathfrak{A}, \alpha)$, where $\alpha$ is the variable assignment that results from the moves of player I. We say that a structure $\left(\mathfrak{B}_{s}, \beta\right)$ is flawless (with 
respect to $\alpha$ ), if $\operatorname{dom}(\beta)=\operatorname{dom}(\alpha)$, and for all $j \in \operatorname{dom}(\alpha)$ and all $r \in\{0,1\}^{n}$, we have

$$
\alpha(j)=a_{r} \Longleftrightarrow \beta(j)=b_{r} .
$$

Note that if $a_{s} \in \operatorname{ran}(\alpha)$, then there is no $\beta$ such that $\left(\mathfrak{B}_{s}, \beta\right)$ is flawless. On the other hand, if $a_{s} \notin \operatorname{ran}(\alpha)$, then there is a unique $\beta$ such that $\left(\mathfrak{B}_{s}, \beta\right)$ is flawless; we denote this $\beta$ by $\beta_{s, \alpha}$.

Furthermore, we say that a structure $\left(\mathfrak{B}_{s}, \beta\right)$ is good enough (with respect to $\alpha$ ), if it is not flawless, but $\operatorname{dom}(\beta)=\operatorname{dom}(\alpha)$, and (10) holds for all $j \in$ $\operatorname{dom}(\alpha)$ and all $r \neq s$, and there is a string $t \in\{0,1\}^{n}$ with $\left|\left\{i: s_{i} \neq t_{i}\right\}\right|=1$ such that

$$
\alpha(j)=a_{s} \Longleftrightarrow \beta(j)=c_{t}
$$

for all $j \in \operatorname{dom}(\alpha)$. Thus, if $a_{s} \in \operatorname{ran}(\alpha)$, then for each $t \in\{0,1\}^{n}$ with $\left|\left\{i: s_{i} \neq t_{i}\right\}\right|=1$ there is a unique $\beta$ such that $\left(\mathfrak{B}_{s}, \beta\right)$ is good enough; we denote this $\beta$ by $\beta_{s, t, \alpha}$.

For the rest of this section, $\mathcal{B}$ will always denote a set of structures of the form $\left(\mathfrak{B}_{s}, \beta\right)$, and $\mathcal{A}$ will denote a singleton set $\{(\mathfrak{A}, \alpha)\}$. The measure of the set $\mathcal{B}$ is defined to be

$$
M(\mathcal{B})=(n+1) \cdot f(\mathcal{B})+g(\mathcal{B})
$$

where $f(\mathcal{B})$ is the number of flawless structures in $\mathcal{B}$ and $g(\mathcal{B})$ is the number of good enough structures in $\mathcal{B}$.

Lemma 14 If $M(\mathcal{B})>1$, then there is no atomic formula $\phi$ such that $(\mathcal{A}, \mathcal{B}) \models \phi$ or $(\mathcal{A}, \mathcal{B}) \models \neg \phi$.

Proof. If $M(\mathcal{B})>1$, then either there is a flawless structure in $\mathcal{B}$, or there are at least two good enough structures in $\mathcal{B}$. If $\left(\mathfrak{B}_{s}, \beta\right) \in \mathcal{B}$ is flawless, then by condition (10), $(\mathfrak{A}, \alpha)$ and $\left(\mathfrak{B}_{s}, \beta\right)$ satisfy the same atomic formulas, whence no atomic formula separates $\mathcal{A}$ and $\mathcal{B}$.

Assume then, that $\left(\mathfrak{B}_{s}, \beta_{s, t, \alpha}\right)$ and $\left(\mathfrak{B}_{s^{\prime}}, \beta_{s^{\prime}, t^{\prime}, \alpha}\right)$ are two distinct good enough structures in $\mathcal{B}$. Let $\phi$ be an atomic formula. If $\phi$ is an identity $x_{j}=x_{k}$ (with $j, k \in \operatorname{dom}(\alpha)$ ), then it follows easily from (10) and (11) that $(\mathfrak{A}, \alpha) \models \phi \Longleftrightarrow\left(\mathfrak{B}_{s}, \beta_{s, t, \alpha}\right) \models \phi$. Thus, $\phi$ does not separate $\mathcal{A}$ and $\mathcal{B}$.

Consider then the case $\phi=P_{l}\left(x_{j}\right)$, where $j \in \operatorname{dom}(\alpha)$. Let $\alpha(j)=a_{r}$. If $r \neq s$, then $\beta_{s, t, \alpha}(j)=b_{r}$, and we have

$$
(\mathfrak{A}, \alpha) \models \phi \Longleftrightarrow r_{l}=1 \Longleftrightarrow\left(\mathfrak{B}_{s}, \beta_{s, t, \alpha}\right) \models \phi .
$$


Similarly, if $r \neq s^{\prime}$, then $(\mathfrak{A}, \alpha) \models \phi \Longleftrightarrow\left(\mathfrak{B}_{s^{\prime}}, \beta_{s^{\prime}, t^{\prime}, \alpha}\right) \models \phi$. Assume finally, that $r=s=s^{\prime}$. Since $\left(\mathfrak{B}_{s}, \beta_{s, t, \alpha}\right) \neq\left(\mathfrak{B}_{s^{\prime}}, \beta_{s^{\prime}, t^{\prime}, \alpha}\right)$, we have $t \neq t^{\prime}$. Moreover, since $\left|\left\{i: r_{i} \neq t_{i}\right\}\right|=\left|\left\{i: r_{i} \neq t_{i}^{\prime}\right\}\right|=1$, either $r_{l}=t_{l}$ or $r_{l}=t_{l}^{\prime}$. Thus, it is not possible that $\phi$ separates $(\mathfrak{A}, \alpha)$ from both $\left(\mathfrak{B}_{s}, \beta_{s, t, \alpha}\right)$ and $\left(\mathfrak{B}_{s^{\prime}}, \beta_{s^{\prime}, t^{\prime}, \alpha}\right)$. We conclude that in all cases $(\mathcal{A}, \mathcal{B}) \not \forall \phi$ and $(\mathcal{A}, \mathcal{B}) \not \forall \neg \phi$ Q.E.D.

Lemma 15 (a) If $\mathcal{B}=\mathcal{C} \cup \mathcal{D}$, then $M(\mathcal{C})+M(\mathcal{D}) \geq M(\mathcal{B})$.

(b) If $\mathcal{A}^{\prime}=\mathcal{A}(F / j)$ and $\mathcal{B}^{\prime}=\mathcal{B}(\star / j)$, then $M\left(\mathcal{B}^{\prime}\right) \geq M(\mathcal{B})-1$.

Proof. (a) If $\mathcal{B}=\mathcal{C} \cup \mathcal{D}$, then obviously $f(\mathcal{C})+f(\mathcal{D}) \geq f(\mathcal{B})$, and $g(\mathcal{C})+$ $g(\mathcal{D}) \geq g(\mathcal{B})$. Hence we have $M(\mathcal{C})+M(\mathcal{D})=(n+1)(f(\mathcal{C})+f(\mathcal{D}))+(g(\mathcal{C})+$ $g(\mathcal{D})) \geq M(\mathcal{B})$.

(b) Let $F((\mathfrak{A}, \alpha))=a_{r}$. Thus, $\mathcal{A}^{\prime}=\left\{\left(\mathfrak{A}, \alpha^{\prime}\right)\right\}$, where $\alpha^{\prime}=\alpha\left(a_{r} / j\right)$. Observe first that if $\left(\mathfrak{B}_{s}, \beta_{s, \alpha}\right)$ is a flawless structure, and $r \neq s$, then $\left(\mathfrak{B}_{s}, \beta_{s, \alpha}\left(b_{r} / j\right)\right)=\left(\mathfrak{B}_{s}, \beta_{s, \alpha^{\prime}}\right)$ is also flawless, and clearly $\left(\mathfrak{B}, \beta_{s, \alpha}\right) \in \mathcal{B} \Longleftrightarrow$ $\left(\mathfrak{B}, \beta_{s, \alpha^{\prime}}\right) \in \mathcal{B}^{\prime}$.

Assume then that $\left(\mathfrak{B}_{s}, \beta_{s, t, \alpha}\right)$ is a good enough structure. If $r \neq s$, then $\left(\mathfrak{B}_{s}, \beta_{s, t, \alpha}\left(b_{r} / j\right)\right)=\left(\mathfrak{B}_{s}, \beta_{s, t, \alpha^{\prime}}\right)$ is also good enough. On the other hand, if $r=s$, then $\left(\mathfrak{B}_{s}, \beta_{s, t, \alpha}\left(c_{t} / j\right)\right)=\left(\mathfrak{B}_{s}, \beta_{s, t, \alpha^{\prime}}\right)$ is good enough. In both cases, $\left(\mathfrak{B}, \beta_{s, t, \alpha}\right) \in \mathcal{B} \Longleftrightarrow\left(\mathfrak{B}, \beta_{s, t, \alpha^{\prime}}\right) \in \mathcal{B}^{\prime}$.

Thus, we see that if $\mathcal{B}$ does not contain a flawless structure of the form $\left(\mathfrak{B}_{r}, \beta_{r, \alpha}\right)$, then $f\left(\mathcal{B}^{\prime}\right)=f(\mathcal{B})$ and $g\left(\mathcal{B}^{\prime}\right)=g(\mathcal{B})$, whence the claim is true.

Assume finally, that there is a flawless structure $\left(\mathfrak{B}_{r}, \beta_{r, \alpha}\right)$ in $\mathcal{B}$. Since $\alpha^{\prime}(j)=a_{r}$, no structure $\left(\mathfrak{B}_{r}, \beta\right)$ is flawless with respect to $\alpha^{\prime}$. On the other hand, for each $t \in\{0,1\}^{n}$ with $\left|\left\{i: r_{i} \neq t_{i}\right\}\right|=1$, there is a new good enough structure $\left(\mathfrak{B}_{r}, \beta_{r, \alpha}\left(c_{t} / j\right)\right)=\left(\mathfrak{B}_{r}, \beta_{r, t, \alpha^{\prime}}\right)$ in $\mathcal{B}^{\prime}$. Thus, in this case $f\left(\mathcal{B}^{\prime}\right)=f(\mathcal{B})-1$ and $g\left(\mathcal{B}^{\prime}\right)=g(\mathcal{B})+n$, whence $M\left(\mathcal{B}^{\prime}\right)=(n+1)(f(\mathcal{B})-$ $1)+g(\mathcal{B})+n=M(\mathcal{B})-1 . \quad$ Q.E.D.

Lemma 16 If $w<M(\mathcal{B})$, then player II has a winning strategy in $\mathrm{EF}_{w}^{\exists}(\mathcal{A}, \mathcal{B})$.

Proof. We prove the claim by induction on $w$. Consider first the case $w=1$. By the definition of the game $\operatorname{EF}_{1}^{\exists}(\mathcal{A}, \mathcal{B})$, there are no moves, and player I wins only if there is an atomic formula $\phi$ such that $(\mathcal{A}, \mathcal{B}) \models \phi$ or $(\mathcal{A}, \mathcal{B})=\neg \phi$. Since $M(\mathcal{B})>w=1$, by Lemma 14, there is no such $\phi$.

Assume then that $w>1$, and the claim is true for all $u<w$. Since $M(\mathcal{B})>w \geq 1$, by Lemma 14 again, player I does not win the game without making moves. Consider then the options of player I for his first move. 
Making a left splitting move $\mathcal{A}=\mathcal{C} \cup \mathcal{D}$ is not possible, since $\mathcal{A}$ is a singleton $\{(\mathfrak{A}, \alpha)\}$. Suppose then that player I makes a right splitting move $w=u+v$ and $\mathcal{B}=\mathcal{C} \cup \mathcal{D}$. Then by Lemma $15(\mathrm{a}), M(\mathcal{C})+M(\mathcal{D}) \geq M(\mathcal{B})$, and since $w<M(\mathcal{B})$, either $u<M(\mathcal{C})$, or $v<M(\mathcal{D})$. If $u<M(\mathcal{C})$, then by induction hypothesis, player II has a winning strategy in the game $\operatorname{EF}_{u}^{\exists}(\mathcal{A}, \mathcal{C})$. Similarly, if $v<M(\mathcal{D})$, then player II has a winning strategy in the game $\operatorname{EF}_{v}^{\exists}(\mathcal{A}, \mathcal{D})$. Thus, by choosing the appropriate position $(u, \mathcal{A}, \mathcal{C})$ or $(v, \mathcal{A}, \mathcal{D})$, player II is guaranteed to win the game $\operatorname{EF}_{w}^{\exists}(\mathcal{A}, \mathcal{B})$.

Suppose then that player I starts with a left supplementing move $j$ and $F$, where $F$ is a choice function for $\mathcal{A}$. The next position in the game is then $\left(w-1, \mathcal{A}^{\prime}, \mathcal{B}^{\prime}\right)$, where $\mathcal{A}^{\prime}=\mathcal{A}(F / j)$ and $\left.\mathcal{B}^{\prime}=\mathcal{B}(\star / j)\right)$. By Lemma 15)(b) and our assumption $w<M(\mathcal{B})$, we have $w-1<M(\mathcal{B})-1 \leq M\left(\mathcal{B}^{\prime}\right)$, whence by induction hypothesis, player II has a winning startegy in the continuation of the game $\operatorname{EF}_{w}^{\exists}(\mathcal{A}, \mathcal{B})$ from position $\left(w-1, \mathcal{A}^{\prime}, \mathcal{B}^{\prime}\right)$ onwards. $\quad$ Q.E.D.

Consider now the classes $\mathcal{A}_{0}$ and $\mathcal{B}_{0}$ defined in the beginning of this section. Since the variable assignment in the only structure in $\mathcal{A}_{0}$ is empty, all the $2^{n}$ structures $\left(\mathfrak{B}_{s}, \emptyset\right)$ in $\mathcal{B}_{0}$ are flawless. Thus, $M\left(\mathcal{B}_{0}\right)=(n+1) f\left(\mathcal{B}_{0}\right)+$ $g\left(\mathcal{B}_{0}\right)=(n+1) 2^{n}$, and by Lemma 16, player II has a winning strategy in the game $\operatorname{EF}_{w}^{\exists}\left(\mathcal{A}_{0}, \mathcal{B}_{0}\right)$ whenever $w<(n+1) 2^{n}$. As all Boolean combinations of the predicates $P_{1}, \ldots, P_{n}$ are non-empty in $\mathfrak{A}$, but each structure in $\mathcal{B}_{0}$ has an empty Boolean combination, we get the desired lower bound result:

Corollary 17 If $\phi$ is an existential first order sentence expressing the property that all Boolean combinations of $n$ unary predicates are non-empty, then the size of $\phi$ is at least $(n+1) 2^{n}$.

Q.E.D.

\section{The Existential Complexity of the Length of Linear Order}

As we noted in the introdution, for each $n$ there is first order sentence $\phi_{n}$ of logarithmic size with respect to $n$ which expresses the property that the length of a linear order is at least $n$. However, the sentence $\phi_{n}$ has an unbounded number of quantifier alternations. In this section we show that $2 n-1$ is the minimum size of an existential sentence expressing this property.

To prove the upper bound, define the following sequence of existential formulas: 


$$
\begin{aligned}
& \psi_{2}:=\exists x_{1} \exists x_{2}\left(x_{1}<x_{2}\right), \quad \text { and } \\
& \psi_{n+1}:=\exists x_{n+1}\left(\psi_{n} \wedge x_{n}<x_{n+1}\right) \quad \text { for all } n \geq 2 .
\end{aligned}
$$

Clearly $\psi_{n}$ is true in a linear order if and only if its length is at least $n$, and an easy induction shows that $\mathrm{w}\left(\psi_{n}\right)=2 n-1$.

To prove the lower bound, we will use again the existential game $\mathrm{EF}_{w}^{\exists}$. Let $\mathcal{A}_{0}=\{(\mathfrak{A}, \emptyset)\}$, where $\mathfrak{A}$ is a linear order of length $n$, and let $\mathcal{B}_{0}=\{(\mathfrak{B}, \emptyset)\}$, where $\mathcal{B}$ is a linear order of length $n-1$. Our aim is to show that player II has a winning strategy in the game $\operatorname{EF}_{w}^{\exists}\left(\mathcal{A}_{0}, \mathcal{B}_{0}\right)$ for all $w<2 n-1$.

Consider a position $(u, \mathcal{A}, \mathcal{B})$ in the game $\operatorname{EF}_{w}^{\exists}\left(\mathcal{A}_{0}, \mathcal{B}_{0}\right)$. Since the game is existential, $\mathcal{A}$ consists of a single structure $(\mathfrak{A}, \alpha)$. Let $a_{1}<^{\mathfrak{A}} \cdots<^{\mathfrak{A}} a_{l}$ be the elements in $\operatorname{ran}(\alpha)$, and let $a_{0}$ and $a_{l+1}$ be the least and the largest element in $\mathfrak{A}$, respectively. We say that a variable assignment $\beta$ in $\mathfrak{B}$ is acceptable (with respect to $\alpha$ ) if $\operatorname{dom}(\beta)=\operatorname{dom}(\alpha)$, there are elements $b_{1} \leq^{\mathfrak{B}} \cdots \leq^{\mathfrak{B}} b_{l}$ such that $\operatorname{ran}(\beta)=\left\{b_{1}, \ldots, b_{l}\right\}$ and for all $i \in\{1, \ldots, l\}$ and all $j \in \operatorname{dom}(\alpha)$

$$
\alpha(j)=a_{i} \Longleftrightarrow \beta(j)=b_{i}
$$

In other words, $\beta$ is acceptable if and only if the mapping $\alpha(j) \mapsto \beta(j)$, $j \in \operatorname{dom}(\alpha)$, preserves the relation $\leq$.

Furthermore, we say that $\beta$ is nice (with respect to $\alpha$ ), if it is acceptable, and in addition $\left|\left\{i \leq l: d\left(a_{i}, a_{i+1}\right) \neq d\left(b_{i}, b_{i+1}\right)\right\}\right|=1$, where $d(x, y)$ is the distance between $x$ and $y$ in the given linear order, and $b_{0}$ and $b_{l+1}$ are the least and the largest element in $\mathfrak{B}$, respectively. The distance from defect of $\beta$ is defined by $\delta(\beta)=d\left(b_{i}, b_{i+1}\right)$, where $i \leq l$ is the unique index such that $d\left(a_{i}, a_{i+1}\right) \neq d\left(b_{i}, b_{i+1}\right)$; we denote this index by $i(\beta)$. Note that $d\left(b_{i}, b_{i+1}\right)=d\left(a_{i}, a_{i+1}\right)-1$ for $i=i(\beta)$. Note also, that for each $i \leq l$ there is exactly one nice $\beta$ such that $i(\beta)=i$.

For the rest of this section, we assume that $\mathcal{A}$ is a singleton set $\{(\mathfrak{A}, \alpha)\}$ and $\mathcal{B}$ is a set of structures of the form $(\mathfrak{B}, \beta)$. The niceness measure of $\mathcal{B}$ is defined to be

$$
N(\mathcal{B})=\sum_{\beta \in \mathcal{N}}(2 \delta(\beta)+1)
$$

where $\mathcal{N}$ is the set of all nice variable assignments $\beta$ such that $(\mathfrak{B}, \beta) \in \mathcal{B}$.

Lemma 18 If $N(\mathcal{B})>1$, then there is no atomic formula $\phi$ such that $(\mathcal{A}, \mathcal{B}) \models \phi$ or $(\mathcal{A}, \mathcal{B}) \models \neg \phi$. 
Proof. If $N(\mathcal{B})>1$, then either there is a nice assignment $\beta \in \mathcal{N}$ such that $\delta(\beta) \geq 1$, or there are two distinct nice assignments $\beta, \beta^{\prime} \in \mathcal{N}$. Assume first that $\beta$ is a nice assignment in $\mathcal{N}$, and $\delta(\beta) \geq 1$. Then there are elements $a_{1}, \ldots, a_{l}, b_{1}, \ldots, b_{l}$ such that $\operatorname{ran}(\alpha)=\left\{a_{1}, \ldots, a_{l}\right\}, \operatorname{ran}(\beta)=\left\{b_{1}, \ldots, b_{l}\right\}$, $a_{1}<^{\mathfrak{A}} \ldots<^{\mathfrak{A}} a_{l}$ and $b_{1} \leq^{\mathfrak{B}} \ldots \leq^{\mathfrak{B}} b_{l}$. Since $\beta$ is nice, $d\left(b_{i}, b_{i+1}\right)=\delta(\beta) \geq 1$ for $i=i(\beta)$, and $d\left(b_{i}, b_{i+1}\right)=d\left(a_{i}, a_{i+1}\right) \geq 1$ for all other $i \leq l$. Thus, we

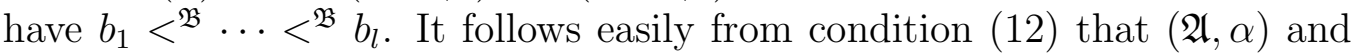
$(\mathfrak{B}, \beta)$ satisfy the same atomic formulas, whence no atomic formula separates $\mathcal{A}$ and $\mathcal{B}$.

Assume then that $\beta, \beta^{\prime} \in \mathcal{N}$, and $\beta \neq \beta^{\prime}$. As noted above, this means that $i(\beta) \neq i\left(\beta^{\prime}\right)$. Let $a_{1}, \ldots, a_{l}$ be as above, and let $\operatorname{ran}(\beta)=\left\{b_{1}, \ldots, b_{l}\right\}$ and $\operatorname{ran}\left(\beta^{\prime}\right)=\left\{b_{1}^{\prime}, \ldots, b_{l}^{\prime}\right\}$ with $b_{1} \leq^{\mathfrak{B}} \ldots \leq^{\mathfrak{B}} b_{l}$ and $b_{1}^{\prime} \leq^{\mathfrak{B}} \ldots \leq^{\mathfrak{B}} b_{l}^{\prime}$. As above, we see that $b_{i}<^{\mathfrak{B}} b_{i+1}$ for all $i \leq l$ except $i=i(\beta)$, and similarly $b_{i}^{\prime}<^{\mathfrak{B}} b_{i+1}^{\prime}$ for all $i \leq l$ except $i=i\left(\beta^{\prime}\right)$. Moreover, since $i(\beta) \neq i\left(\beta^{\prime}\right)$, for all $i, j \leq l$, we have either $\left(a_{i}<^{\mathfrak{A}} a_{j} \Longleftrightarrow b_{i}<^{\mathfrak{B}} b_{j}\right)$ or $\left(a_{i}<^{\mathfrak{A}} a_{j} \Longleftrightarrow b_{i}^{\prime}<^{\mathfrak{B}} b_{j}^{\prime}\right)$. Using condition (12), it is now easy to see that no atomic formula separates the sets $\mathcal{A}$ and $\mathcal{B}$.

Q.E.D.

Lemma 19 (a) If $\mathcal{B}=\mathcal{C} \cup \mathcal{D}$, then $N(\mathcal{C})+N(\mathcal{D}) \geq N(\mathcal{B})$

(b) If $\mathcal{A}^{\prime}=\mathcal{A}(F / j)$ and $\mathcal{B}^{\prime}=\mathcal{B}(\star / j)$, then $N\left(\mathcal{B}^{\prime}\right) \geq N(\mathcal{B})-1$.

Proof. (a) Assume that $\mathcal{B}=\mathcal{C} \cup \mathcal{D}$. Let $\mathcal{N}, \mathcal{O}$ and $\mathcal{P}$ be the sets of nice assignments $\beta$ such that $(\mathfrak{B}, \beta) \in \mathcal{B},(\mathfrak{B}, \beta) \in \mathcal{C}$ and $(\mathfrak{B}, \beta) \in \mathcal{D}$, respectively. Then we have

$$
\begin{aligned}
N(\mathcal{B}) & =\sum_{\beta \in \mathcal{N}}(2 \delta(\beta)+1) \\
& \leq \sum_{\beta \in \mathcal{O}}(2 \delta(\beta)+1)+\sum_{\beta \in \mathcal{P}}(2 \delta(\beta)+1)=N(\mathcal{C})+N(\mathcal{D}) .
\end{aligned}
$$

(b) Let $\mathcal{N}$ and $\mathcal{N}^{\prime}$ be the sets of nice $\beta$ such that $(\mathfrak{B}, \beta)$ is in $\mathcal{B}$ and $\mathcal{B}^{\prime}$, respectively. Let $\operatorname{ran}(\alpha)=\left\{a_{1}, \ldots, a_{l}\right\}$ with $a_{1}<^{\mathfrak{A}} \ldots<^{\mathfrak{A}} a_{l}$, where $\alpha$ is the assignment such that $\mathcal{A}=\{(\mathfrak{A}, \alpha)\}$. As usual, we denote by $a_{0}$ and $a_{l+1}$ the least and the largest element in $\mathfrak{A}$, respectively. Furthermore, we denote $F((\mathfrak{A}, \alpha))$ by $c$.

Assume first that $c=\alpha(k)$ for some $k \in \operatorname{dom}(\alpha)$. It is easy to see that for any assignment $\beta$,

$$
\beta \in \mathcal{N} \Longleftrightarrow \beta(d / j) \in \mathcal{N}^{\prime},
$$


where $d=\beta(k)$, and moreover $\delta(\beta(d / j))=\delta(\beta)$. Note also that if $\beta \neq \beta^{\prime}$, then $\beta(d / j) \neq \beta^{\prime}\left(d^{\prime} / j\right)$. Thus, in this case we have $N\left(\mathcal{B}^{\prime}\right)=N(\mathcal{B})$.

Assume next that $c=a_{0}$ or $c=a_{l+1}$. Then as above, we see that $\beta \in \mathcal{N} \Longleftrightarrow \beta(d / j) \in \mathcal{N}^{\prime}$, where $d$ is the least or the largest element in $\mathcal{B}$, respectively, and $\delta(\beta(d / j))=\delta(\beta)$. Thus, also in this case we conclude that $N\left(\mathcal{B}^{\prime}\right)=N(\mathcal{B})$.

Assume finally, that $c \notin\left\{a_{0}, \ldots, a_{l+1}\right\}$. Then there is an index $h \leq l$ such that $a_{h}<^{\mathfrak{A}} c<^{\mathfrak{A}} a_{h+1}$. Let $\beta$ be a nice assignment in $\mathcal{N}$, and let $\operatorname{ran}(\beta)=\left\{b_{1}, \ldots, b_{l}\right\}$ with $b_{1} \leq^{\mathfrak{B}} \cdots \leq^{\mathfrak{B}} b_{l}$. As noted earlier, there is exactly one nice assignment $\beta_{h}$ such that $i\left(\beta_{h}\right)=h$. If $\beta \neq \beta_{h}$, then $d\left(b_{h}, b_{h+1}\right)=$ $d\left(a_{h}, a_{h+1}\right)$, whence there is an element $d$ such that $d\left(b_{h}, d\right)=d\left(a_{h}, c\right)$ and $d\left(d, b_{h+1}\right)=d\left(c, a_{h+1}\right)$. This means that $\delta(\beta(d / j))=\delta(\beta)$, and clearly $\beta(d / j)$ is in $\mathcal{N}^{\prime}$.

On the other hand, if $\beta=\beta_{h}$, then $d\left(b_{h}, b_{h+1}\right)=d\left(a_{h}, a_{h+1}\right)-1$, and there are elements $d$ and $e$ such that $d\left(b_{h}, d\right)=d\left(a_{h}, c\right)-1, d\left(d, b_{h+1}\right)=d\left(c, a_{h+1}\right)$, $d\left(b_{h}, e\right)=d\left(a_{h}, c\right)$ and $d\left(e, b_{h+1}\right)=d\left(c, a_{h+1}\right)-1$. Let $\beta^{\prime}=\beta_{h}(d / j)$ and $\beta^{\prime \prime}=\beta_{h}(e / j)$. Then $\beta^{\prime}, \beta^{\prime \prime} \in \mathcal{N}^{\prime}$, and we have

$$
\delta\left(\beta^{\prime}\right)+\delta\left(\beta^{\prime \prime}\right)=d\left(b_{h}, d\right)+d\left(e, b_{h+1}\right)=d\left(a_{h}, a_{h+1}\right)-2=\delta\left(\beta_{h}\right)-1,
$$

whence

$$
\left(2 \delta\left(\beta^{\prime}\right)+1\right)+\left(2 \delta\left(\beta^{\prime \prime}\right)+1\right)=2\left(\delta\left(\beta_{h}\right)-1\right)+2=\left(2 \delta\left(\beta_{h}\right)+1\right)-1 .
$$

Thus, if $\beta_{h}$ is in $\mathcal{N}$, we get

$$
\begin{aligned}
N\left(\mathcal{B}^{\prime}\right) & =\sum_{\beta^{\prime} \in \mathcal{N}^{\prime}}\left(2 \delta\left(\beta^{\prime}\right)+1\right) \\
& =\sum_{\beta \in \mathcal{N} \backslash\left\{\beta_{h}\right\}}(2 \delta(\beta)+1)+\left(\left(2 \delta\left(\beta_{h}\right)+1\right)-1\right)=N(\mathcal{B})-1,
\end{aligned}
$$

and if $\beta_{h}$ is not in $\mathcal{N}$, we have $N\left(\mathcal{B}^{\prime}\right)=N(\mathcal{B})$.

Q.E.D.

Lemma 20 If $w<N(\mathcal{B})$, then player II has a winning strategy in $\mathrm{EF}_{w}^{\exists}(\mathcal{A}, \mathcal{B})$.

Proof. The proof is verbatim the same as for Lemma 16, just replace $M(\mathcal{B})$ by $N(\mathcal{B})$.

Q.E.D.

The proof of the lower bound result is also similar to that in the previous section. Consider the sets $\mathcal{A}_{0}=\{(\mathfrak{A}, \emptyset)\}$ and $\mathcal{B}_{0}=\{(\mathfrak{B}, \emptyset)\}$. Clearly $\delta(\emptyset)=$ 
$n-1$, and so $N\left(\mathcal{B}_{0}\right)=2(n-1)+1=2 n-1$. Thus, by Lemma 20, player II has a winning strategy in the game $\operatorname{EF}_{w}^{\exists}\left(\mathcal{A}_{0}, \mathcal{B}_{0}\right)$ for all $w<2 n-1$. Since the length of the linear order $\mathfrak{A}$ is $n$, while the length of $\mathfrak{B}$ is less than $n$, we have proved

Corollary 21 If $\phi$ is an existential first order sentence expressing the property that the length of a linear order is at least $n$, then the size of $\phi$ is at least $2 n-1$.

Q.E.D.

\section{References}

[1] Micah Adler and Neil Immerman. An $n$ ! lower bound on formula size. ACM Trans. Comput. Log., 4(3):296-314, 2003. 16th Annual Symposium on Logic in Computer Science, 2001 (Boston, MA).

[2] M. Ajtai. $\Sigma_{1}^{1}$-formulae on finite structures. Ann. Pure Appl. Logic, 24(1):1-48, 1983.

[3] Merrick Furst, James B. Saxe, and Michael Sipser. Parity, circuits, and the polynomial-time hierarchy. Math. Systems Theory, 17(1):13-27, 1984.

[4] Martin Grohe and Nicole Schweikardt. The succinctness of first-order logic on linear orders. Log. Methods Comput. Sci., 1(1):1:6, 25, 2005.

[5] Jouko Väänänen. On the semantics of informational independence. Log. J. IGPL, 10(3):339-352, 2002.

[6] Ingo Wegener. The complexity of Boolean functions. Wiley-Teubner Series in Computer Science. John Wiley \& Sons Ltd., Chichester, 1987. 